\title{
Altered contractile properties of the quadriceps muscle in people with spinal cord injury following functional electrical stimulated cycle training
}

\author{
HL Gerrits*,1,2, A de Haan ${ }^{1,3}$, AJ Sargeant ${ }^{1,3}$, A Dallmeijer ${ }^{1}$ and MTE Hopman ${ }^{2}$ \\ ${ }^{1}$ Institute for Fundamental and Clinical Human Movement Sciences, Vrije University Amsterdam, Amsterdam, The \\ Netherlands; ${ }^{2}$ Institute for Fundamental and Clinical Human Movement Sciences, University of Nijmegen, Nijmegen, \\ The Netherlands; ${ }^{3}$ Neuromuscular Biology Group, Manchester Metropolitan University, Alsager, UK
}

\begin{abstract}
Study design: A longitudinal training study.
Objectives: To assess if contractile speed and fatigability of paralysed quadriceps muscles in individuals with spinal cord injury (SCI) can be altered by functional electrical stimulation leg cycle ergometry (FES-LCE) training.

Settings: The Sint Maartenskliniek rehabilitation centre and the University of Nijmegen, Nijmegen, the Netherlands.

Methods: Contractile properties of the quadriceps muscle were studied in seven people with motor-complete SCI who participated in a FES-LCE training program. Subjects trained for 30 min, three times per week for 6 weeks. Contractile speed and fatigue characteristics of electrically stimulated isometric contractions were compared before and after 6 weeks of FESLCE.

Results: Fatigue resistance improved following FES-LCE training as indicated by the higher forces maintained in response to repetitive electrical stimulation. In contrast with an improved fatigue resistance, the maximal rate of force rise was unaffected, the speed of relaxation increased and the fusion of a $10 \mathrm{~Hz}$ force signal decreased. Furthermore, the force-frequency relationship shifted to the right at low stimulation frequencies, indicated by a decline in the ratio of 1 and $100 \mathrm{~Hz}$ force responses as well as the ratio of 10 and $100 \mathrm{~Hz}$ force responses. Conclusion: FES-LCE training can change the physiological properties of the quadriceps muscle in people with SCI. Even after a short period of training, the stimulated muscles become more resistant to fatigue. Furthermore, the increased speed of relaxation and associated decreased fusion and altered force-frequency relationship following training may be related to adaptations in the calcium handling processes, which reflect an early response of long-term disused muscles.
\end{abstract}

Spinal Cord (2000) 38, 214-223

Keywords: cycle ergometer; muscle characteristics; fatigue; paraplegia

\section{Introduction}

Spinal cord injury (SCI) results in loss of motor control, sensation and autonomic function below the level of the spinal lesion. This traumatic event often leads to significant central and peripheral physiological adaptations including impaired cardiovascular fitness, ${ }^{1}$ muscle atrophy ${ }^{2,3}$ with concomitant greater prevalence of fast type II muscle fibres, ${ }^{4-6}$ osteoporosis ${ }^{7,8}$ and reduced blood circulation of the lower limbs. ${ }^{9-11}$

For almost two decades now, much attention has been focused on improving health and fitness of individuals with SCI using a functional electrical stimulation leg cycle ergometer (FES-LCE). This ergometer provides

*Correspondence: HL Gerrits, Institute for Fundamental and Clinical Human Movement Sciences, Vrije University Amsterdam, v/d Boechorststraat 9, 1081 BT Amsterdam, The Netherlands computerised electrical stimulation to hamstring, gluteal and quadriceps muscles in a co-ordinated sequence, which enables active leg cycling. ${ }^{12}$ Several studies that assessed effects of FES-LCE training have demonstrated an improved cycling performance,,${ }^{3,13-17}$ and improved cardiovascular fitness. ${ }^{15}$ Musculoskeletal adaptations in the lower limbs following FES-LCE included reduced loss of bone mineral density, ${ }^{18}$ increased muscle mass ${ }^{3,14,19}$ and proportional increases in capillary number. ${ }^{19}$ Furthermore, it has recently been demonstrated that FES-LCE training can significantly alter the expression of myosin heavy chain in the quadriceps muscle of people with SCI. The cycle training induced a change from a predominance of muscle fibres containing IIB or a mixture of IIA/IIB isoforms to a predominance of fibres containing only the IIA isoform. ${ }^{3,20}$ 
From animal studies, it is well known that chronically increased contractile activity can lead to adaptations in the muscle fibre composition and associated physiological properties of the muscle, such as an increased resistance to fatigue and altered contractile characteristics. ${ }^{21,22}$ In agreement with these findings, studies that applied different modes of electrical stimulation protocols on paralysed muscles of people with SCI, reported a decreased number of glycolytic type IIB and an increased number of oxidative type IIA ${ }^{2,23,24}$ or type $\mathrm{I}^{2}$ fibres. Furthermore, in the tibialis anterior muscle, these structural, molecular and metabolic changes were accompanied by an increased fatigue resistance and slowing of contractions. ${ }^{25}$

Recently, we have shown that the quadriceps muscles of individuals with SCI are characterised by faster contraction and relaxation rates and increased fatigability compared with muscles of able-bodied individuals. ${ }^{26}$ Although these muscles have been studied extensively with respect to structural, morphological properties, to our knowledge no systemic data are available in the literature, which quantify alterations in the contractile properties of the stimulated quadriceps muscles after FES-LCE training. It may be expected, however, that these physiological properties undergo significant adaptations that are consistent with alterations in fibre type expression. $^{3,20}$ Therefore, it is hypothesised that the characteristics of the quadriceps muscles in people with SCI shift towards that of more fatigue resistant slower muscles following FES-LCE training. To test this hypothesis we assessed the effect of a 6-week FESLCE training period on the contractile properties of the paralysed quadriceps muscle of individuals with SCI.

\section{Methods}

\section{Subjects}

This study included seven male subjects with motorcomplete spinal lesions. Table 1 represents the characteristics of the subjects with respect to the lesion and body composition. All subjects participated in a pre-training screening procedure. Screening included a general physical examination, performed by the rehabilitation physician; evaluation of joint range of motion, spinal reflexes and spasticity performed by the local physiotherapist. Subjects who met any of the following exclusion criteria were excluded from participation: cardiac arrhythmia, high blood pressure (systolic blood pressure $>180 \mathrm{mmHg}$, diastolic blood pressure $>110 \mathrm{mmHg}$ ), presence of a pacemaker, pressure ulcers, metal implants in the area of stimulation, severely reduced mobility in hip or knee joints, absence of spinal reflexes, and previous bone fractures. All subjects were carefully informed about training and testing procedures and involved risks and gave their written informed consent. The Medical Ethical Committee of the University of Nijmegen approved this study.

\section{FES-LCE training}

The cycle training was performed using a computer controlled FES leg cycle ergometer (Ergys 2, Therapeutic Alliances Inc., Ohio, USA). This device provides electrical stimulation to 12 self-adhesive $50 \times 89 \mathrm{~mm}$ surface electrodes (Bioflex, PE 3590, Danica, Leusden, the Netherlands), placed over hamstring, gluteal and quadriceps muscles (two electrodes for each muscle). Electrical stimulation (monophasic square wave pulses of $450 \mu \mathrm{s}$ duration, $30 \mathrm{~Hz}$, synchronised for each pair of electrodes) is applied in a co-ordinated sequence allowing cyclic patterns of muscle contractions resulting in leg cycling. The device is programmed to increase the stimulation current, with a maximum of $140 \mathrm{~mA}$, in order to achieve a targetpedalling rate of $50 \mathrm{rpm}$. The external pedal resistance of the ergometer can be varied with $1 / 8 \mathrm{kp}$ increments (corresponding with $\sim 6.1 \mathrm{~W}$ at $50 \mathrm{rpm}$ ). When the pedalling rate drops below $45 \mathrm{rpm}$, the resistance is reduced and when the pedalling rate drops below $35 \mathrm{rpm}$, the stimulation is stopped.

\section{Training protocol}

All subjects trained for 6 weeks, three times a week. A training session consisted of 30 min FES-LCE exercise. Each exercise session started with $1 \mathrm{~min}$ of passive

Table 1 Characteristics of SCI subjects with respect to body composition and the level, classification and duration of the lesion

\begin{tabular}{|c|c|c|c|c|c|c|}
\hline Subject & $\begin{array}{c}\text { Age } \\
\text { (years) }\end{array}$ & $\begin{array}{l}\text { Height } \\
(m)\end{array}$ & $\begin{array}{c}\text { Weight } \\
\text { (kg) }\end{array}$ & $\begin{array}{c}\text { Lesion } \\
\text { level }\end{array}$ & $\begin{array}{l}\text { ASIA } \\
\text { class }^{*}\end{array}$ & $\begin{array}{l}\text { Lesion duration } \\
\text { (years) }\end{array}$ \\
\hline 1 & 44 & 1.91 & 95 & $\mathrm{C} 5 / \mathrm{C} 6$ & B & 14 \\
\hline 2 & 36 & 1.72 & 62 & C5 & A & 18 \\
\hline 3 & 30 & 1.78 & 68 & T4 & A & 2 \\
\hline 4 & 61 & 1.80 & 82 & $\mathrm{~T} 7 / \mathrm{T} 8$ & A & 2 \\
\hline 5 & 40 & 1.78 & 78 & T8 & A & 5 \\
\hline 6 & 44 & 1.85 & 88 & C5 & B & 27 \\
\hline 7 & 28 & 1.89 & 72 & $\mathrm{~T} 4$ & A & 1 \\
\hline
\end{tabular}

*ASIS (American Spinal Injury Association ${ }^{49}$ ) score is used to classify the completeness of the lesion: A, sensory and motor complete; B, sensory incomplete but motor complete) 
warming-up (without stimulation) as a research assistant turned the crankshaft manually. After the warming-up, stimulation of the leg muscles started, allowing active FES-LCE training until the subject could no longer maintain the pedalling rate above $35 \mathrm{rpm}$ (represents one 'run'). When fatigue occurred before the end of the 30-min training session this run was followed by a 5-min rest period. This procedure was repeated with a maximum of five runs available to complete $30 \mathrm{~min}$ of active cycling. Each bout of exercise was followed by a 2-min passive cooling down, during which the pedals were turned by the assistant. When a subject was able to complete $30 \mathrm{~min}$ of FES-LCE exercise in one run with at least $90 \%$ of the programmed pedal resistance, the resistance was increased with $1 / 8 \mathrm{kp}$ in the next training session up to a maximum of $7 / 8 \mathrm{kp}$.

\section{Thigh girth}

Thigh circumference was measured at $5 \mathrm{~cm}$ above the superior aspect of the patella and at mid-thigh ( $\sim 20 \mathrm{~cm}$ above the patella) before and after the training period. In order to optimise the reproducibility of these measurements the average of three measurements was taken at each location.

\section{Measurements of contractile properties}

Isometric contractions of the knee extensors were obtained while subjects were seated upright with the pelvis and upper thigh securely fixed to the seat and the knee angle fixed at $100^{\circ}$. Electrically stimulated quadriceps contractions were generated using a high voltage constant current stimulator (model DS7A, Digitimer Limited, Hertfordshire, UK), delivering electrical pulses of $0.2 \mathrm{~ms}$ width to surface electrodes $(10 \times 13 \mathrm{~cm}$, Electro-Medical Supplies, Greenham Limited, UK) placed over the anterior thigh. The muscles were activated with a stimulation intensity of $121 \pm 30 \mathrm{~mA}$. Although we were not able to determine exactly what proportion of the muscle was being stimulated, it was established from previous experiments that approximately $25 \%$ to $30 \%$ of the muscle can be activated with stimulation intensities between $120-160 \mathrm{~mA}^{26}$

Some of the subjects developed muscle spasms during stimulation with intensities that exceeded $120 \mathrm{~mA}$. In these cases, lower stimulation intensities were chosen. Binder-Macleod et $a l^{27}$ showed little systematic change in contractile properties with variable stimulation intensities. We used the same stimulation current in the pre- and post-training experiments for each individual and therefore it can be assumed that the intensity of the stimulation current did not materially affect the results of the present study.

Knee extension forces were recorded using a force transducer connected to a non-extensible strap placed around the lower leg. Electrical trains of $1 \mathrm{~s}$ duration were applied to the muscle at stimulation frequencies of $1,10,20,50$ and $100 \mathrm{~Hz}$ with a rest period of $2 \mathrm{~min}$ between the stimulation trains.

The resistance to fatigue was assessed by activating the quadriceps muscle repetitively for 2 min using 30$\mathrm{Hz}$ stimulation trains of $1 \mathrm{~s}$ duration every $2 \mathrm{~s}$ (ie on: off time $=1 \mathrm{~s}: 1 \mathrm{~s})$. To assess the recovery of the muscle, 1-s stimulation trains of $100 \mathrm{~Hz}$ were applied at 0,2 and $5 \mathrm{~min}$ post-exercise.

In order to minimise possible effects of muscle temperature before and after training, room temperature was kept constant at $25^{\circ} \mathrm{C}$ during the testing

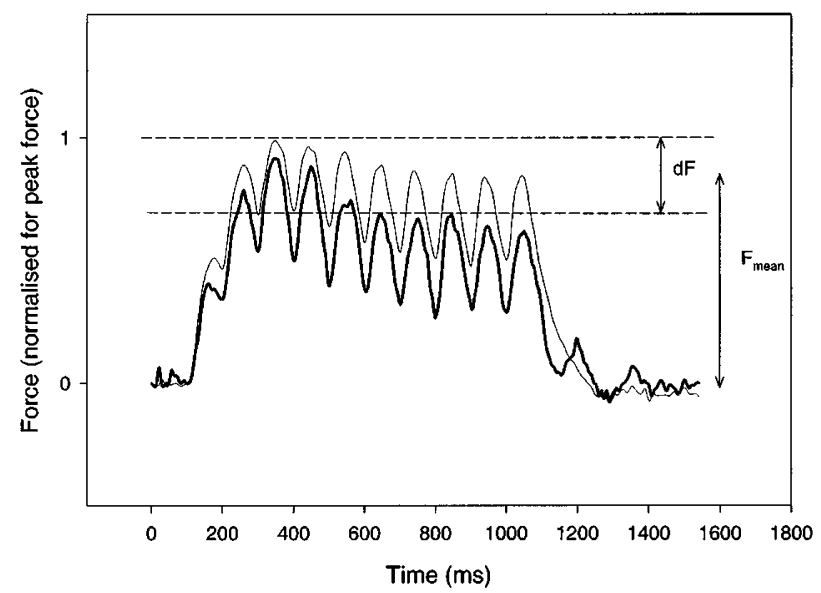

Figure 1 Typical example of a $10-\mathrm{Hz}$ force response before (thin line) and after (bold line) 6 weeks FES-LCE training. Forces are normalised for peak force. dF Represents the average force of three consecutive force oscillations just after the peak force is reached and $F_{\text {mean }}$ represents the force during this time

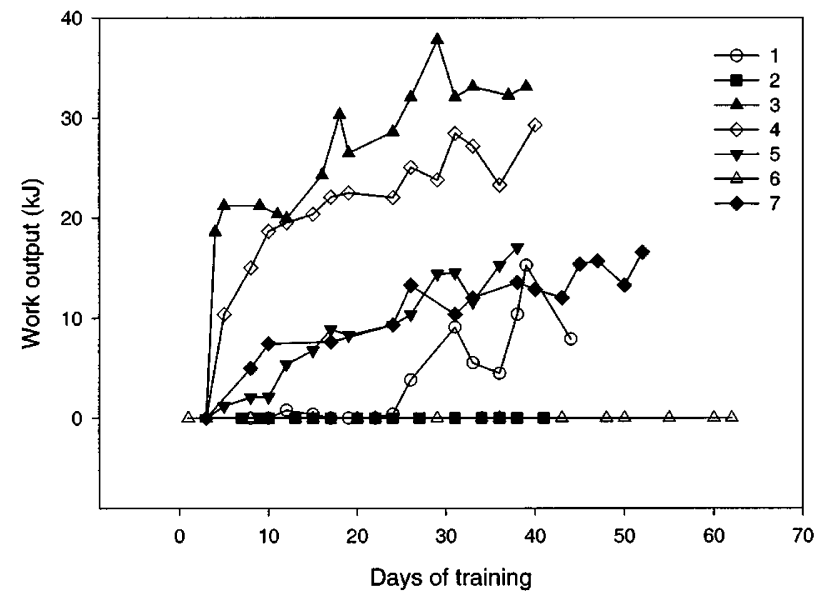

Figure 2 Progression in work output during the FES-LCE training program for seven individuals with SCI. During three training sessions, exercise test protocols were performed on the ergometer, which differed from the original training protocol. Work output values of these sessions are therefore not presented in this graph 
procedure and subjects were in the room at least $45 \mathrm{~min}$ before the actual protocol started.

\section{Data analysis}

Off-line analysis of force records was performed using custom-made software programs. Several indices of contractile speed were obtained. Normalised maximal rate of force rise (MFR) was calculated from the positive filtered $(30 \mathrm{~Hz}$ filter frequency) force differential and expressed as a percentage of peak force according to the method described by Buller et al. ${ }^{28}$ Half relaxation time $(1 / 2 \mathrm{Rt})$, which is related to cross-

(a)

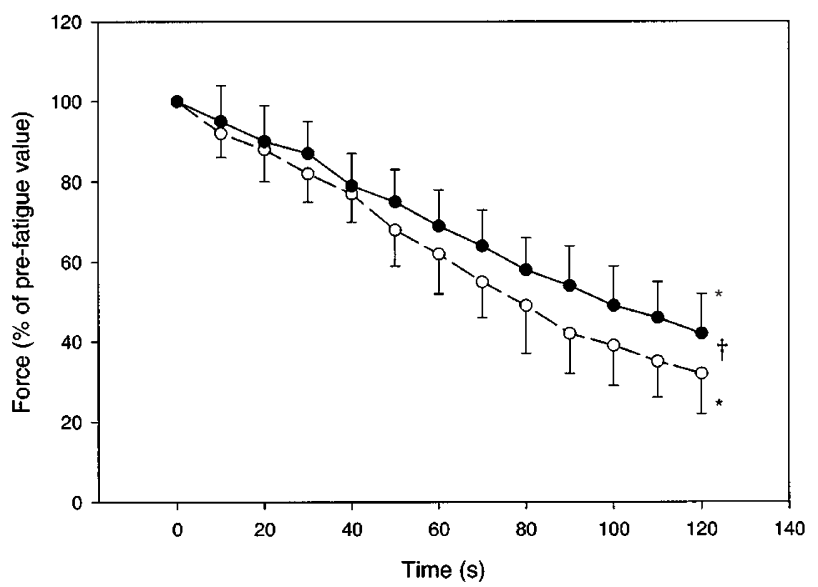

(b)

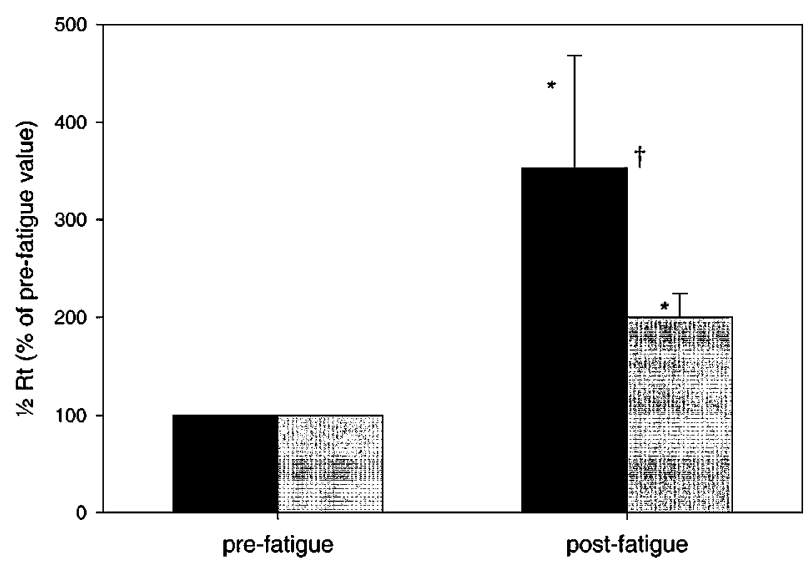

Figure 3 Force (a) and half relaxation time (1/2 Rt) (b) expressed as percent of the pre-fatigue value during $2 \mathrm{~min}$ of repeated $30-\mathrm{Hz}$ stimulation. Force responses before (open circles, dashed line) and after (closed circles, solid line) 6 weeks of FES-LCE training are plotted every $10 \mathrm{~s}$. 1/2 Rt values before (black bars) and after (grey bars) training are given of the first (pre-fatigue) and the last (post-fatigue) contraction. Values are mean $\pm \mathrm{SD}, n=7$ (a), $n=6$ (b). Missing values are due to stimulus artefacts. *Refers to significantly different from pre-fatigue value, $P<0.05$. $\dagger$ Refers to significantly different from pre-training value, $P<0.05$ bridge dissociation and calcium re-uptake, ${ }^{29}$ was defined as the time taken for force to decline from $50 \%$ to $25 \%$ of the peak $100-\mathrm{Hz}$ force. The degree of fusion as a result of $10 \mathrm{~Hz}$ stimulation was determined by calculating the force oscillation amplitudes (FOA) relative to the mean force according to the method described by Gerrits et al. ${ }^{26}$ This was established by taking the average of the three consecutive oscillation amplitudes in the $10-\mathrm{Hz}$ tetanus after force reached its peak value, divided by the mean force during that time (Figure 1).

In order to compare force-frequency relationships, which can distinguish fast-twitch from slow-twitch muscles, ${ }^{30}$ ratios of 1 and $100-\mathrm{Hz}$ force responses and of 10 and $100-\mathrm{Hz}$ force responses were calculated before and after training.

Force responses resulting from $30-\mathrm{Hz}$ stimulation were continuously recorded during the fatigue proto-

(a)

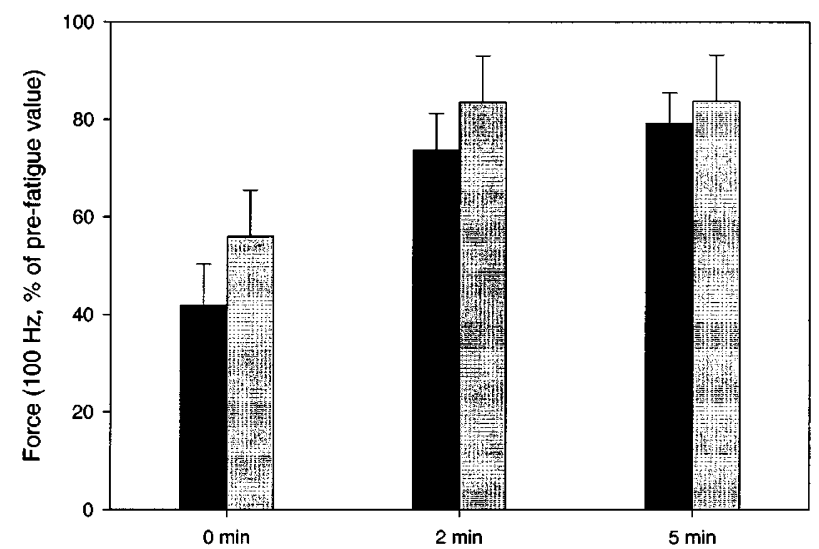

(b)

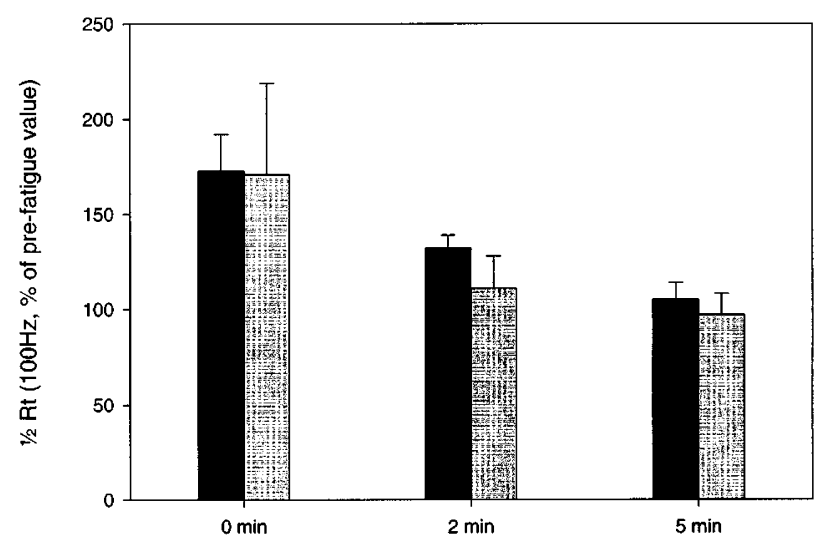

Figure 4 Force (a) and half relaxation time (1/2 Rt) (b) of $100-\mathrm{Hz}$ responses during recovery from the fatigue protocol before (black bars) and after (grey bars) 6 weeks FES-LCE training. Values at $0 \mathrm{~min}, 2 \mathrm{~min}$ and $5 \mathrm{~min}$ post-exercise are expressed as per cent of the pre-fatigue values. Values are mean $+\mathrm{SD} \quad(n=7(\mathrm{a}), n=6(\mathrm{~b}))$. Missing values are due to stimulus artefacts. Recovery of force or $1 / 2 \mathrm{Rt}$ was not significantly different after training 
(a)

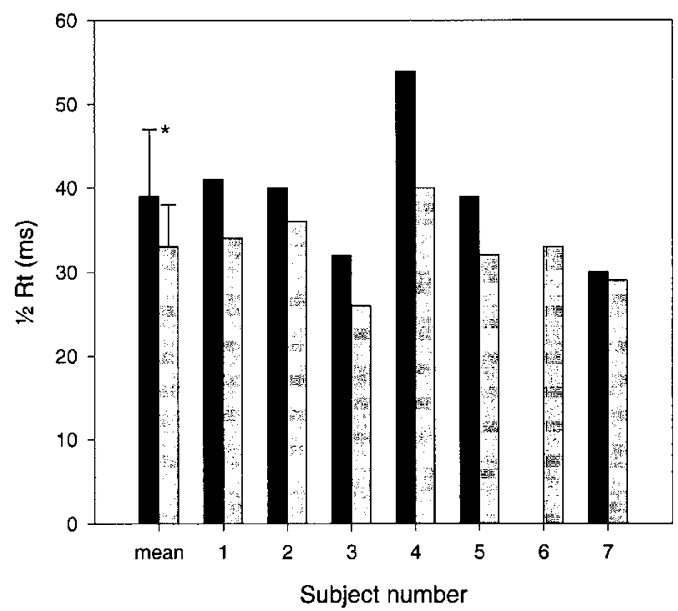

(c)

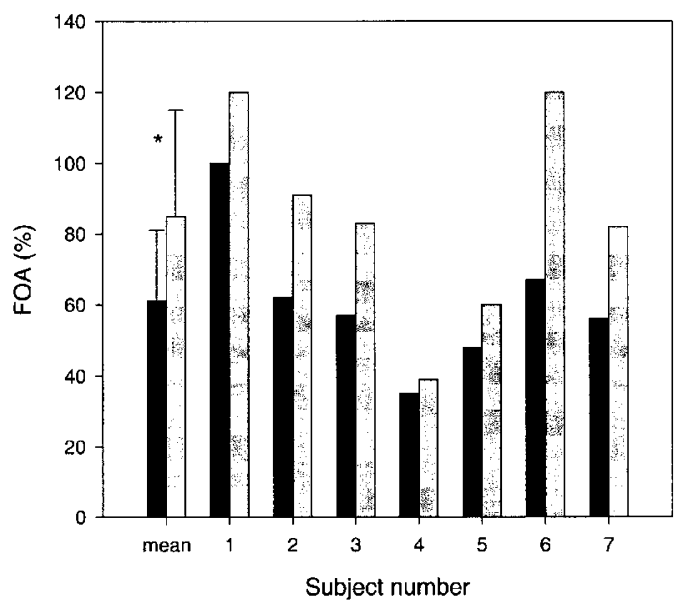

(b)

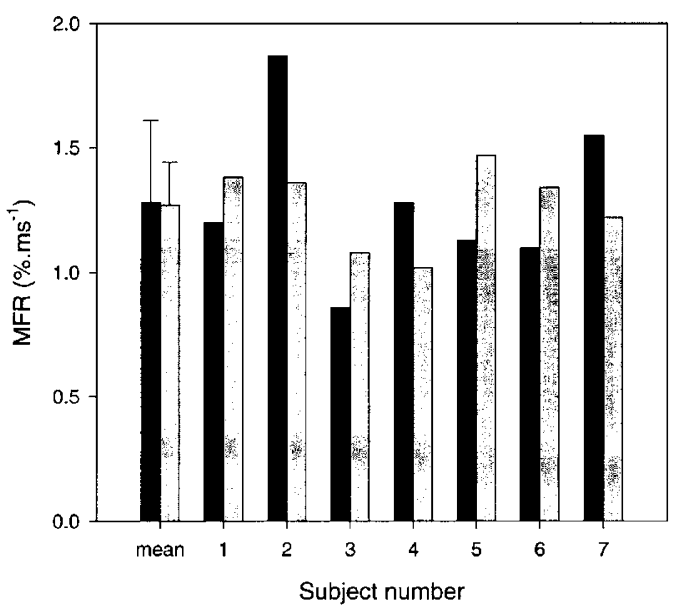

(d)

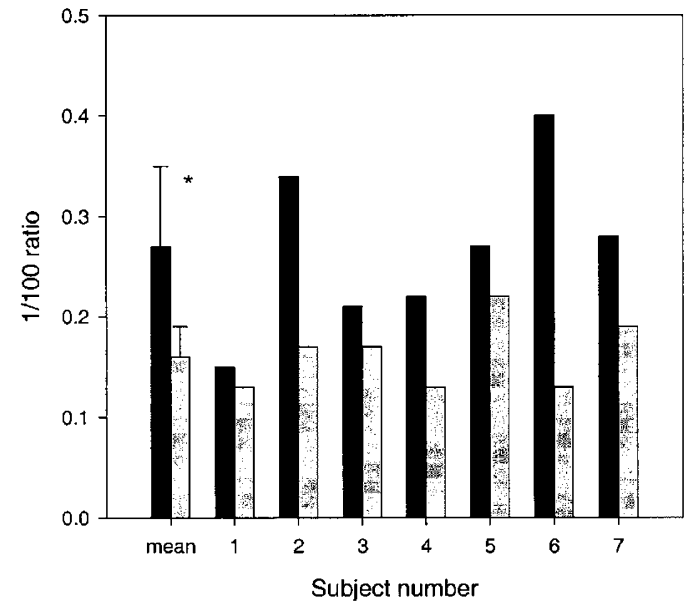

(e)

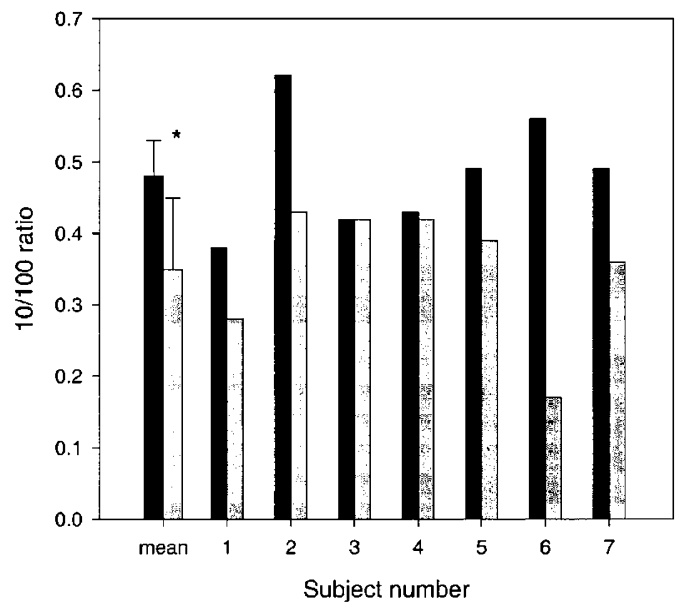

Figure 5 Contractile properties before (black bars) and after (grey bars) training. Individual data of (a) half relaxation time ( $1 / 2$ $\mathrm{Rt}$ ), (b) maximal rate of force rise (MFR), (c) relative force oscillation amplitudes (FOA), (d) ratio of 1 and $100-\mathrm{Hz}$ responses (1/100 ratio), and (e) ratio of 10 and $100-\mathrm{Hz}$ responses (10/100 ratio) from subjects 1 to 7 . Mean values \pm SD are also shown. Missing values are due to stimulus artefacts. ${ }^{*}$ Refers to significantly different from pre-training values, $P<0.05$ 
col. To compare differences in fatigue induced force decline and slowing of relaxation, force and $1 / 2 \mathrm{Rt}$ of the $30-\mathrm{Hz}$ responses were calculated at the start and at 2 min of the fatigue protocol. In order to characterise the recovery of the muscle, force and $1 / 2$ Rt from 100 $\mathrm{Hz}$ responses were calculated at $2 \mathrm{~min}$ and $5 \mathrm{~min}$ after the fatigue protocol.

\section{Statistical analysis}

Comparisons of contractile properties before and after FES-LCE training were made using paired $t$-tests. A repeated measures analysis of variance was used to compare the recovery of force and $1 / 2 \mathrm{Rt}$, before and after FES-LCE training. All values are described as mean $\pm S D$, unless otherwise stated and levels of significance for two-tailed tests were set at 0.05 .

\section{Results}

\section{FES-LCE exercise training}

All subjects completed the total number of 18 training sessions. The majority of the subjects accomplished these sessions within 6 weeks. Two subjects completed the training in longer periods: subject no. 6 in $\sim 9$ weeks and subject no. 7 in $\sim 7.5$ weeks. At the start of the training period, most subjects had a very low exercise tolerance to FES-LCE: the mean work output, averaged over the first three training sessions, was $4 \pm 5 \mathrm{~kJ}$. Most subjects showed an increase in work output as training progressed, although a large variability between subjects was found. After 6 weeks of training, work output, averaged over the last three training sessions, had significantly improved to $14 \pm 13 \mathrm{~kJ}$, respectively $(P=0.019)$. The changes in

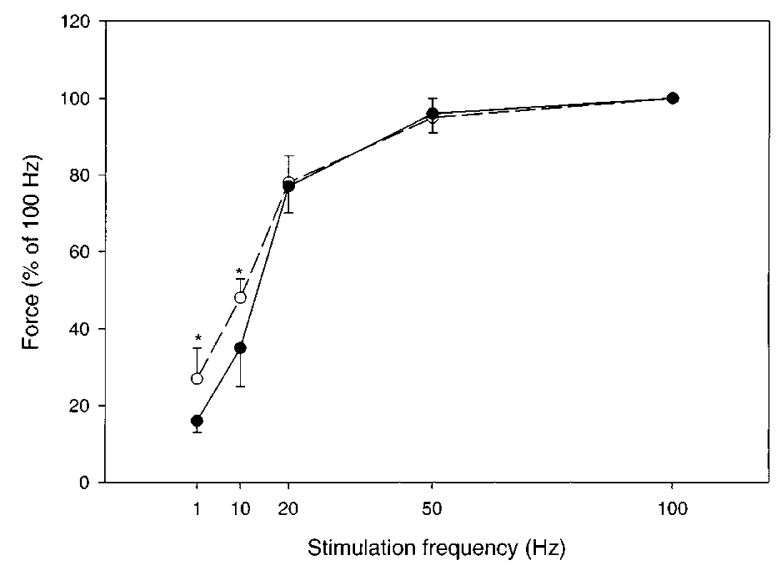

Figure 6 Force-frequency relationship before (open circles, dotted line) and after (closed circles, solid line) 6 weeks of FES-LCE training. Force responses to different stimulation frequencies are normalised for peak isometric $100-\mathrm{Hz}$ force. Values are mean $\pm \mathrm{SD}, n=7$. $*$ Refers to significantly different from pre-training values, $P<0.05$ total work output $(\mathrm{kJ})$ for each subject achieved during the training period is shown in Figure 2. Work output in subject 2 and 6 did not increase during the 6 weeks of FES-LCE. Their total exercise time however, improved from $\sim 9$ and $7 \mathrm{~min}$, respectively, before training (average of the first three training sessions) to $\sim 21$ and $13 \mathrm{~min}$, respectively, after training (average of the last three training sessions).

\section{Fatigue and recovery}

Expressed as a percentage of the pre-fatigue value, force responses generated during repeated $30-\mathrm{Hz}$ stimulation declined progressively during the fatigue protocol before and after training (Figure 3a). There was a large variability in fatigue resistance between subjects and this was negatively correlated with the time since injury (Pearson correlation coefficient: $-0.87, P=0.01)$. However, quadriceps force declined significantly less $(P=0.012)$ following 6 weeks of FESLCE. At the end of the 2-min stimulation protocol, force declined to $32 \pm 10 \%$ of the pre-fatigue value before training and to $42 \pm 10 \%$ after training.

Following $2 \mathrm{~min}$ of repeated stimulation, 1/2 Rt increased to $353 \pm 115 \%$ of the pre-fatigue value before the FES-LCE training. After the training, the slowing of relaxation was significantly reduced $(200 \pm 24 \%$ of the pre-fatigue value, $P=0.015)$ (Figure 3b). Figure 4 presents the recovery of force (a) and $1 / 2$ Rt (b) resulting from $100-\mathrm{Hz}$ responses after the 2-min fatigue protocol.

The relative changes in both force and $1 / 2 \mathrm{Rt}$ during recovery were similar before and after 6 weeks of FESLCE training $(P=0.266$ and 0.545 , respectively).

\section{Contractile properties: contractile speed and low frequency fusion}

Figure 5 presents the contractile characteristics before and after 6 weeks FES-LCE training. MFR remained unchanged after training $\left(1.28 \pm 0.33 \% . \mathrm{ms}^{-1}\right.$ before and $1.27 \pm 0.17 \% . \mathrm{ms}^{-1}$ after the training program, respectively). However, mean $1 / 2$ Rt decreased significantly $(P=0.014)$ from $39 \pm 8 \mathrm{~ms}$ before the FES-LCE training program to $33 \pm 8 \mathrm{~ms}$ after 6 weeks of FESLCE training. Furthermore, the degree of fusion in a $10-\mathrm{Hz}$ force response diminished, which was represented by a significant increase in FOA from $61 \pm 20 \%$ before to $85 \pm 30 \%$ after FES-LCE training $(P=\overline{0.006})$.

\section{Contractile properties: frequency response}

The normalised peak force responses, generated at each frequency before and after 6 weeks of FES-LCE training are presented in Figure 6. As can be clearly seen the force-frequency curve shifted to the right at low stimulation frequencies following training. This shift is indicated by a significant decline in the ratio of 1 and $100-\mathrm{Hz}$ responses from $0.27 \pm 0.08$ to $0.16 \pm 0.03$ $(P=0.018)$, and a significant decline in the ratio of 10 
and $100-\mathrm{Hz}$ responses from $0.48 \pm 0.05$ to $0.35 \pm 0.10$ $(P=0.038)$ after the training procedure (see also Figure $5 \mathrm{~d}$ and e, respectively).

\section{Thigh circumference}

Six weeks of FES-LCE training did not significantly alter thigh circumference. Thigh girth measured $5 \mathrm{~cm}$ proximally of the upper aspect of the patella was $40.2 \pm 3.2 \mathrm{~cm}$ before, and $40.4 \pm 2.5 \mathrm{~cm}$ after the training period. Mid-thigh circumference was $47.8 \pm 2.9 \mathrm{~cm}$ before, and $48.0 \pm 3.4 \mathrm{~cm}$ after the training.

\section{Discussion}

This study was undertaken to quantify changes in the functional characteristics of the quadriceps muscles in individuals with SCI as a result of a 6-week FEScycling program. This is the first study to demonstrate profound alterations in selected isometric contractile speed properties of the paralysed quadriceps muscle following FES-LCE training. Training increased the fatigue resistance of the quadriceps muscles but to our surprise, contractile speed characteristics adapted towards those of faster muscles.

At the beginning of the training period, most SCI subjects had a very low exercise tolerance during FESLCE and demonstrated a low resistance to fatigue during $2 \mathrm{~min}$ of repeated stimulation. The close negative correlation found between the per cent of pre-fatigue force that remained after the 2-min fatigue protocol and the time since injury occurred, indicates that the fatigue resistance of these muscles decreased with the duration of disuse, which may explain the variation in muscle endurance between subjects. This study clearly provided evidence that fatigue resistance can be improved after only 6 weeks of FES-LCE training since all subjects showed a reduction in the rate at which force declined and a reduction in the slowing of relaxation in response to $2 \mathrm{~min}$ of repeated stimulation after the training period. Despite the heterogeneity of the study population, all subjects showed the same changes, indicating that FES-LCE training can improve muscle endurance capacity in a broad population with a large variety of characteristics. Recovery of force and relaxation rate after the 2-min fatigue protocol seemed not affected by training, but the lack of more frequent force measurements during the recovery period limits the interpretation of these results. Martin et $a l^{2}$ reported higher activities of oxidative enzymes after prolonged electrical stimulation of the tibialis anterior muscle in people with SCI. Furthermore, Chilibeck et $a l^{19}$ found an increase in the capillary number in the quadriceps muscle of subjects with SCI after 8 weeks of FES-LCE. These results indicate that electrical stimulation training programs can increase the oxidative capacity and improve the blood supply to the exercising muscle, which would improve the delivery of oxygen and energy to the exercising muscles and diminish accumulation of metabolic products. These adaptations are known to be the earliest changes after chronic electrical stimulation $^{31}$ and most likely underlie the improved endurance capacity of the quadriceps muscle in the present study.

In addition, the improved cycle performance, which was also found in previous studies ${ }^{3,13-17}$ is in agreement with the increased endurance capacity of these muscles. Cycle performance is probably best represented by an increased work output, which is determined by the level of pedal resistance multiplied by the time that resistance could be maintained. Two subjects were unable to increase their pedal resistance above $0 \mathrm{kp}$ during the 6 -week training program, and thus work output had not improved in these people. However, the total time these individuals were able to cycle doubled after FES-LCE, indicating a substantial improvement in exercise performance.

It is possible that an increased muscle mass as a result of FES-LCE training contributes to the improved work capacity in some of our subjects. We were not able to demonstrate significant increases in thigh girth, which may have been due to the low sensitivity of these measurements, short exercise period or changes in the relative amount of fat tissue. Other investigators however, using computer tomography, MRI, or muscle biopsies, reported an increased whole muscle $^{3,14}$ or fibre cross sectional area ${ }^{19}$ after a prolonged period, ${ }^{3}$ but also after only 8 weeks ${ }^{14,19}$ of FES-LCE training.

The most striking result of the present study is that 6 weeks of FES-LCE increases the speed of relaxation, consequently leading to a decreased fusion of a $10-\mathrm{Hz}$ force response. As relaxation rate increases, force at low frequencies becomes less fused and tetanic fusion would require higher stimulation frequencies resulting in the right-shift of the force-frequency relationship observed in the present study. The increased relaxation rate appears to be inconsistent with our observation of an increased fatigue resistance and in conflict with findings in numerous previous studies. Three decades ago Salmons and Vrbova ${ }^{32}$ already demonstrated that a transformation of fast- into slowtwitch muscle can be brought about by the application of chronic electrical stimulation in animals and this was confirmed extensively by others. $^{21,22}$ Furthermore, studies that applied electrical stimulation programs on the muscles of humans with SCI, have reported the expected slowing of contractile properties ${ }^{25,33}$ and associated changes in muscle fibre composition towards more oxidative type IIA or type I muscle fibres. ${ }^{2,3,20,23,24,33}$

The speed of relaxation is closely related to the cross-bridge dissociation and the re-uptake of calcium by the sarcoplasmic reticulum. ${ }^{29}$ In animals, chronic low frequency electrical stimulation programs reduce the calcium uptake capacity by decreasing the calcium ATPase activity and these changes would be associated 
with increases in time-to-peak of isometric tension and half relaxation times. ${ }^{34}$ The faster relaxation after training found in the present study is in contrast with these findings, and suggests that FES-LCE increased the calcium uptake in the stimulated quadriceps muscle. To our knowledge, it is unknown how longterm SCI in humans affect the mechanisms involving calcium handling. Hunter et $a l^{35}$ however, reported that the rate of calcium uptake by the sarcoplasmic reticulum was lower in elderly women compared with young women, and that resistance training increased this rate of calcium uptake in elderly women, but not in young women. It is possible therefore, that although normally, fast-twitch muscles have higher rates of calcium uptake than slow-twitch muscles, ${ }^{36}$ these processes may be impaired in the fast-twitch paralysed muscles of people with SCI, owing to long periods of inactivity. Previous studies support this speculation by reporting evidence that implies a reduced rate of calcium uptake by the sarcoplasmic reticulum following denervation, ${ }^{37-39}$ although others have found conflicting results. ${ }^{40,41}$ Thus, we would suggest that following prolonged disuse leading to an impairment of calcium handling mechanisms, training initially leads to an increase in the calcium uptake capacity as reflected by the faster relaxation.

In contrast with the changes in the relaxation phase of the muscle, the rate of force development in a $100-\mathrm{Hz}$ force response remained unchanged, suggesting that 6 weeks of FES-LCE training did not affect the net rate of actomyosin cross-bridge attachment. $^{42}$ It should however be noted that higher stimulation frequencies are needed to generate maximal rate of force rise than to produce maximal tetanic tension. ${ }^{28,43}$ Therefore, stimulation frequencies in excess of $100 \mathrm{~Hz}$ may have been required to obtain the maximal rate of force development. However, the use of higher stimulation frequencies may increase the risk for damage to the weak paralysed muscles and tendons, and we therefore decided not to use stimulation frequencies above $100 \mathrm{~Hz}$. Moreover, it may be expected that differences in the rate of force development as a result of an increased net cross-bridge attachment would be detected already at sub-maximal levels of activation. A more likely explanation for the unchanged rate of force rise is that although adaptations in metabolic properties, capillary blood flow and calcium ATPase activity occur soon after the start of electrical stimulation programs, alterations in myosin ATPase and associated increased rate of tension development develop at a later stage. $^{31,34}$

The increased fatigue resistance, faster speed of relaxation and unchanged rate of force development demonstrated in the current study, probably reflect the first adaptations of long-term disused muscles to electrically induced increases in contractile activity.
The quadriceps muscle in our study was stimulated for $30 \mathrm{~min}$, three times a week, for 6 weeks. Other studies however, who actually reported significant adaptations in structural, molecular and physiological properties towards characteristics of slower, more oxidative muscles used considerably longer or more intensive training programs. Andersen et $a l^{20}$ and Mohr et $a l^{3}$ used a similar FES-LCE training program, but continued the training for 6 to 12 months. Others applied daily electrical stimulation for $1 \mathrm{~h}$ a day during approximately 13 weeks ${ }^{23}$ or even up to $8 \mathrm{~h}$ a day during $6^{33}$ or 24 weeks. ${ }^{2}$ Rochester et $a l^{24,25}$ used a 4-week training program, which was less than in our study, but again stimulation was applied daily for approximately $2.5 \mathrm{~h}$ a day. Thus, it may be speculated that longer or more intensive training periods are required to induce muscular adaptations, which would lead to a detectable slowing of contractile speed characteristics in paralysed muscle of people with SCI.

In addition to altered physiological characteristics of the contractile elements of the muscle, possible mechanical adaptations in the non-contractile tissue of the muscle and/or tendon as a result of training may affect the functional properties of the muscle. Immobilisation leads to an increased muscle stiffness in animals, ${ }^{44,45}$ which may be attributed to both quantitative and qualitative changes in the connective tissue. ${ }^{44}$ Furthermore, contractile activity seems to be important for the maintenance of normal muscle compliance $^{46}$ and training can decrease muscle stiffness. ${ }^{47}$ In contrast, Douglas et $a l^{48}$ who studied muscle tone in humans, reported a decreased muscle stiffness in people with paraplegia compared with noninjured controls. Furthermore, the muscle stiffness of these paralysed muscles was increased by electrically stimulated shortening contractions and decreased by a period of passive movements.

It remains unclear therefore, whether the FES-LCE training in the present study induced an increase or decrease in muscle stiffness and to what extent these possible mechanical adaptations in muscle and tendon may have affected the results of the present study.

The present study clearly demonstrates that 6 weeks of FES-LCE training can improve the physiological properties of the quadriceps muscle in individuals with SCI with respect to fatigue resistance. Furthermore, an increase in the speed of relaxation was found which might reflect an early response in the calcium handling mechanisms of the muscles consequent upon training of long-term disused muscles. The possible impairment of calcium handling following long-term disuse in humans, and the recovery from that impairment is clearly an issue which deserves further investigation if monitoring of training programs is used to inform the development of effective interventions in this group of SCI individuals. It is possible that longer training programs are required to induce significant adaptations towards characteristics of slower contracting muscles. 


\section{References}

1 Davis GM. Exercise capacity of individuals with paraplegia. Med Sci Sports Exerc 1993; 25: 423-432.

2 Martin TP, Stein RB, Hoeppner PH, Reid DC. Influence of electrical stimulation on the morphological and metabolic properties of paralyzed muscle. J Appl Physiol 1992; 72: 1401 1406.

3 Mohr T et al. Long-term adaptation to electrically induced cycle training in severe spinal cord injured individuals [published erratum appears in Spinal Cord 1997 Apr;35(4):262]. Spinal Cord 1997; 35: $1-16$.

4 Burnham $\mathrm{R}$ et al. Skeletal muscle fibre type transformation following spinal cord injury. Spinal Cord 1997; 35: 86-91.

5 Grimby G, Broberg C, Krotkiewska I, Krotkiewski M. Muscle fiber composition in patients with traumatic cord lesion. Scand J Rehabil Med 1976; 8: $37-42$.

6 Lotta $\mathrm{S}$ et al. Morphometric and neurophysiological analysis of skeletal muscle in paraplegic patients with traumatic cord lesion. Paraplegia 1991; 29: 247-252.

7 Biering-Sorensen F, Bohr H, Schaadt O. Bone mineral content of the lumbar spine and lower extremities years after spinal cord lesion. Paraplegia 1988; 26: 293 -301.

8 Ragnarsson KT, Sell GH. Lower extremity fractures after spinal cord injury: a retrospective study. Arch Phys Med Rehabil 1981; 62: $418-423$.

9 Hopman MT, van Asten WN, Oeseburg B. Changes in blood flow in the common femoral artery related to inactivity and muscle atrophy in individuals with long-standing paraplegia. Adv Exp Med Biol 1996; 388: 379-383.

10 Nash MS, Montalvo BM, Applegate B. Lower extremity blood flow and responses to occlusion ischemia differ in exercise-trained and sedentary tetraplegic persons. Arch Phys Med Rehabil 1996; 77: $1260-1265$

11 Taylor PN et al. Limb blood flow, cardiac output and quadriceps muscle bulk following spinal cord injury and the effect of training for the Odstock functional electrical stimulation standing system. Paraplegia 1993; 31: $303-310$.

12 Petrofsky JS, Heaton HHd, Phillips CA. Leg exerciser for training of paralysed muscle by closed-loop control. Med Biol Eng Comput 1984; 22: 298-303.

13 Bremner LA et al. A clinical exercise system for paraplegics using functional electrical stimulation. Paraplegia 1992; 30: 647-655.

14 Hjeltnes $\mathrm{N}$ et al. Improved body composition after $8 \mathrm{wk}$ of electrically stimulated leg cycling in tetraplegic patients. $\mathrm{Am} \mathrm{J}$ Physiol 1997; 273: R1072-1079.

15 Faghri PD, Glaser RM, Figoni SF. Functional electrical stimulation leg cycle ergometer exercise: training effects on cardiorespiratory responses of spinal cord injured subjects at rest and during submaximal exercise. Arch Phys Med Rehabil 1992; 73: $1085-1093$.

16 Ragnarsson KT. Physiologic effects of functional electrical stimulation-induced exercises in spinal cord-injured individuals. Clin Orthopaed Relat Res 1988; 233: 53-63.

17 Sloan KE et al. Musculoskeletal effects of an electrical stimulation induced cycling programme in the spinal injured. Paraplegia 1994; 32: 407-415.

18 Hangartner TN, Rodgers MM, Glaser RM, Barre PS. Tibial bone density loss in spinal cord injured patients: effects of FES exercise. J Rehabil Res Dev 1994; 31: 50-61.

19 Chilibeck PD et al. Histochemical changes in muscles of individuals with spinal cord injury following functional electrical stimulated exercise training. Spinal Cord 1999; 37: $264-268$

20 Andersen JL et al. Myosin heavy chain isoform transformation in single fibres from $\mathrm{m}$. vastus lateralis in spinal cord injured individuals: effects of long-term functional electrical stimulation (FES). Pflugers Archiv Eur J Physiol 1996; 431: 513-518.

21 Pette D, Vrbova G. Adaptation of mammalian skeletal muscle fibers to chronic electrical stimulation. Rev Physiol Biochem Pharmacol 1992; 120: 115-202.
22 Salmons S. Exercise, stimulation and type transformation of skeletal muscle. Int J Sports Med 1994; 15: 136 - 141.

23 Greve JM et al. Functional electrical stimulation (FES): muscle histochemical analysis. Paraplegia 1993; 31: 764-770.

24 Rochester L et al. Influence of electrical stimulation of the tibialis anterior muscle in paraplegic subjects. 2. Morphological and histochemical properties. Paraplegia 1995; 33: 514-522.

25 Rochester L et al. Influence of electrical stimulation of the tibialis anterior muscle in paraplegic subjects. 1. Contractile properties. Paraplegia 1995; 33: 437-449.

26 Gerrits HL et al. Contractile properties of the quadriceps muscle in individuals with spinal cord injury. Muscle Nerve 1999; 22: $1249-1256$.

27 Binder-Macleod SA, Halden EE, Jungles KA. Effects of stimulation intensity on the physiological responses of human motor units. Med Sci Sports Exerc 1995; 27: 556-565.

28 Buller AJ, Lewis DM. The rate of tension development in isometric tetanic contractions of mammalian fast and slow skeletal muscle. J Physiol 1965; 176: $337-354$.

29 Edwards RH, Hill DK, Jones DA. Metabolic changes associated with the slowing of relaxation in fatigued mouse muscle. $J$ Physiol 1975; 251: $287-301$.

30 Cooper S, Eccles JC. The isometric responses of mammalian muscles. J Physiol 1930; 69: 377-385.

31 Salmons S, Henriksson J. The adaptive response of skeletal muscle to increased use. Muscle Nerve 1981; 4: 94-105.

32 Salmons S, Vrbova G. The influence of activity on some contractile characteristics of mammalian fast and slow muscles. J Physiol 1969; 201: 535 - 549.

33 Stein RB et al. Optimal stimulation of paralyzed muscle after human spinal cord injury. $J$ Appl Physiol 1992; 72: 1393-1400.

34 Pette D. JB Wolffe memorial lecture. Activity-induced fast to slow transitions in mammalian muscle. Med Sci Sports Exerc 1984; 16: $517-528$.

35 Hunter SK et al. Human skeletal sarcoplasmic reticulum Ca2+ uptake and muscle function with aging and strength training. $J$ Appl Physiol 1999; 86: $1858-1865$.

36 Pette D, Staron RS. Cellular and molecular diversities of mammalian skeletal muscle fibers. Rev Physiol Biochem Pharmacol 1990; 116: 1 -76.

37 Howell S, Zhan WZ, Sieck GC. Diaphragm disuse reduces Ca2 + uptake capacity of sarcoplasmic reticulum. J Appl Physiol 1997; 82: $164-171$

38 Lehotsky J, Bezakova G, Kaplan P, Raeymaekers L. Distribution of $\mathrm{Ca}(2+)$-modulating proteins in sarcoplasmic reticulum membranes after denervation. Gen Physiol Biophys 1993; 12: $339-348$.

39 Schulte L et al. Sarcoplasmic reticulum Ca2 + pump expression in denervated skeletal muscle. Am J Physiol 1994; 267: C617622 .

40 Sreter FA. Effect of denervation on fragmented sarcoplasmic reticulum of white and red muscle. Exp Neurol 1970; 29: 52 - 64 .

41 Takekura H, Kasuga N, Kitada K, Yoshioka T. Morphological changes in the triads and sarcoplasmic reticulum of rat slow and fast muscle fibres following denervation and immobilization. $J$ Muscle Res Cell Motil 1996; 17: 391-400.

42 Ranatunga KW. Temperature-dependence of shortening velocity and rate of isometric tension development in rat skeletal muscle. J Physiol 1982; 329: 465-483.

43 de Haan A. The influence of stimulation frequency on forcevelocity characteristics of in situ rat medial gastrocnemius muscle. Exp Physiol 1998; 83: 77-84.

44 Williams PE, Goldspink G. Connective tissue changes in immobilised muscle. J Anatomy 1984; 138: 343 - 350 .

45 Gillette PD, Fell RD. Passive tension in rat hindlimb during suspension unloading and recovery: muscle/joint contributions. $J$ Appl Physiol 1996; 81: $724-730$.

46 Williams PE, Catanese T, Lucey EG, Goldspink G. The importance of stretch and contractile activity in the prevention of connective tissue accumulation in muscle. J Anatomy 1988; 158: $109-114$ 
47 Pousson M, Perot C, Goubel F. Stiffness changes and fibre type transitions in rat soleus muscle produced by jumping training. Pflugers Archiv Eur J Physiol 1991; 419: 127-130.

48 Douglas AJ et al. The effects of neuromuscular stimulation on muscle tone at the knee in paraplegia. Exp Physiol 1991; 76: $357-$ 367.
49 Maynard Jr FM et al. International standards for neurological and functional classification of spinal cord injury. American Spinal Injury Association. Spinal Cord 1997; 35: 266-274. 